\title{
ON WEAK-OPEN COMPACT IMAGES OF METRIC SPACES
}

\author{
ZHIYUN YIN
}

Received 2 August 2005; Revised 29 June 2006; Accepted 9 July 2006

We give some characterizations of weak-open compact images of metric spaces.

Copyright (c) 2006 Hindawi Publishing Corporation. All rights reserved.

\section{Introduction and definitions}

To find internal characterizations of certain images of metric spaces is one of central problems in general topology. Arhangel'skiu [1] showed that a space is an open compact image of a metric space if and only if it has a development consisting of point-finite open covers, and some characterizations for certain quotient compact images of metric spaces are obtained in $[3,5,8]$. Recently, Xia [12] introduced the concept of weak-open mappings. By using it, certain $g$-first countable spaces are characterized as images of metric spaces under various weak-open mappings. Furthermore, Li and Lin in [4] proved that a space is $g$-metrizable if and only if it is a weak-open $\sigma$-image of a metric space.

The purpose of this paper is to give some characterizations of weak-open compact images of metric spaces, which showed that a space is a weak-open compact image of a metric space if and only if it has a weak development consisting of point-finite cs-covers.

In this paper, all spaces are Hausdorff, all mappings are continuous and surjective. $\mathbb{N}$ denotes the set of all natural numbers. $\tau(X)$ denotes the topology on a space $X$. For the usual product space $\prod_{i \in \mathbb{N}} X_{i}, \pi_{i}$ denotes the projection $\prod_{i \in \mathbb{N}} X_{i}$ onto $X_{i}$. For a sequence $\left\{x_{n}\right\}$ in $X$, denote $\left\langle x_{n}\right\rangle=\left\{x_{n}: n \in \mathbb{N}\right\}$.

Definition $1.1[1]$. Let $\mathscr{P}=\bigcup\left\{\mathscr{P}_{x}: x \in X\right\}$ be a collection of subsets of a space $X . \mathscr{P}$ is called a weak base for $X$ if

(1) for each $x \in X, \mathscr{P}_{x}$ is a network of $x$ in $X$,

(2) if $U, V \in \mathscr{P}_{x}$, then $W \subset U \cap V$ for some $W \in \mathscr{P}_{x}$,

(3) $G \subset X$ is open in $X$ if and only if for each $x \in G$, there exists $P \in \mathscr{P}_{x}$ such that $P \subset G$.

$\mathscr{P}_{x}$ is called a weak neighborhood base of $x$ in $X$, every element of $\mathscr{P}_{x}$ is called a weak neighborhood of $x$ in $X$. 
Definition 1.2. Let $f: X \rightarrow Y$ be a mapping.

(1) $f$ is called a weak-open mapping [12], if there exists a weak base $\mathscr{B}=\cup\left\{\mathscr{B}_{y}: y \in\right.$ $Y$ \} for $Y$, and for each $y \in Y$, there exists $x_{y} \in f^{-1}(y)$ satisfying the following condition: for each open neighborhood $U$ of $x_{y}, B_{y} \subset f(U)$ for some $B_{y} \in \mathscr{B}_{y}$.

(2) $f$ is called a compact mapping, if $f^{-1}(y)$ is compact in $X$ for each $y \in Y$.

It is easy to check that a weak-open mapping is quotient.

Definition 1.3 [2]. Let $X$ be a space, and $P \subset X$. Then the following hold.

(1) A sequence $\left\{x_{n}\right\}$ in $X$ is called eventually in $P$, if the $\left\{x_{n}\right\}$ converges to $x$, and there exists $m \in \mathbb{N}$ such that $\{x\} \cup\left\{x_{n}: n \geq m\right\} \subset P$.

(2) $P$ is called a sequential neighborhood of $x$ in $X$, if whenever a sequence $\left\{x_{n}\right\}$ in $X$ converges to $x$, then $\left\{x_{n}\right\}$ is eventually in $P$.

(3) $P$ is called sequential open in $X$, if $P$ is a sequential neighborhood at each of its points.

(4) $X$ is called a sequential space, if any sequential open subset of $X$ is open in $X$.

Definition 1.4 [7]. Let $\mathscr{P}$ be a cover of a space $X$.

(1) $\mathscr{P}$ is called a $c s$-cover for $X$, if every convergent sequence in $X$ is eventually in some element of $\mathscr{P}$.

(2) $\mathscr{P}$ is called an $s n$-cover for $X$, if every element of $\mathscr{P}$ is a sequential neighborhood of some point in $X$, and for any $x \in X$, there exists a sequential neighborhood $P$ of $x$ in $X$ such that $P \in \mathscr{P}$.

Definition 1.5 [7]. Let $\left\{\mathscr{P}_{n}\right\}$ be a sequence of covers of a space $X$.

(1) $\left\{\mathscr{P}_{n}\right\}$ is called a point-star network for $X$, if for each $x \in X,\left\langle\operatorname{st}\left(x, \mathscr{P}_{n}\right)\right\rangle$ is a network of $x$ in $X$.

(2) $\left\{\mathscr{P}_{n}\right\}$ is called a weak development for $X$, if for each $x \in X,\left\langle\operatorname{st}\left(x, \mathscr{P}_{n}\right)\right\rangle$ is a weak neighborhood base for $X$.

\section{Results}

THEOREM 2.1. The following are equivalent for a space $X$.

(1) $X$ is a weak-open compact image of a metric space.

(2) X has a weak development consisting of point-finite cs-covers.

(3) $X$ has a weak development consisting of point-finite sn-covers.

Proof. (1) $\Rightarrow(2)$. Suppose that $f: M \rightarrow X$ is a weak-open compact mapping with $M$ a metric space. Let $\left\{{\iota_{n}}_{n}\right\}$ be a sequence consisting of locally finite open covers of $M$ such that $u_{n+1}$ is a refinement of $u_{n}$ and $\left\langle s t\left(K, \cup_{n}\right)\right\rangle$ forms a neighborhood base of $K$ in $M$ for each compact subset $K$ of $M$ (see [7, Theorem 1.3.1]). For each $n \in \mathbb{N}$, put $\mathscr{P}_{n}=f\left(\cup_{n}\right)$. Since $f$ is compact, then $\left\{\mathscr{P}_{n}\right\}$ is a point-finite cover sequence of $X$.

If $x \in V$ with $V$ open in $X$, then $f^{-1}(x) \subset f^{-1}(V)$. Since $f^{-1}(x)$ compact in $M$, then $\operatorname{st}\left(f^{-1}(x), \mathcal{U}_{n}\right) \subset f^{-1}(V)$ for some $n \in \mathbb{N}$, and so $\operatorname{st}\left(x, \mathscr{P}_{n}\right) \subset V$. Hence $\left\langle\operatorname{st}\left(x, \mathscr{P}_{n}\right)\right\rangle$ forms a network of $x$ in $X$. Therefore, $\left\{\mathscr{P}_{n}\right\}$ is a point-star network for $X$.

We will prove that every $\mathscr{P}_{k}$ is a $c s$-cover for $X$. Since $f$ is weak-open, there exists a weak base $\mathscr{B}=\cup\left\{\mathscr{S}_{x}: x \in X\right\}$ for $X$, and for each $x \in X$, there exists $m_{x} \in f^{-1}(x)$ 
satisfying the following condition: for each open neighborhood $U$ of $m_{x}$ in $M, B \subset f(U)$ for some $B \in \mathscr{B}_{x}$.

For each $x \in X$ and $k \in \mathbb{N}$, let $\left\{x_{n}\right\}$ be a sequence converging to a point $x \in X$. Take $U \in U_{k}$ with $m_{x} \in U$. Then $B \subset f(U)$ for some $B \in \mathscr{B}_{x}$. Since $B$ is a weak neighborhood of $x$ in $X$, then $B$ is a sequential neighborhood of $x$ in $X$ by [6, Corollary 1.6.18], so $f(U) \in \mathscr{P}_{k}$ is also. Thus $\left\{x_{n}\right\}$ is eventually in $f(U)$. This implies that each $\mathscr{P}_{k}$ is a cs-cover for $X$. Since $f(U)$ is a sequential neighborhood of $x$ in $X$, then $\operatorname{st}\left(x, \mathscr{P}_{k}\right)$ is also. Obviously, $X$ is a sequential space. So $\left\langle s t\left(x, \mathscr{P}_{k}\right)\right\rangle$ is a weak neighborhood base of $x$ in $X$.

In words, $\left\{\mathscr{P}_{n}\right\}$ is a weak development consisting of point-finite $c s$-covers for $X$.

$(2) \Rightarrow(3)$. By Theorem A in [5], $X$ is a sequential space. It suffices to prove that if $\mathscr{P}$ is a point-finite $c s$-cover for $X$, then some subset of $\mathscr{P}$ is an $s n$-cover for $X$. For each $x \in X$, denote $(\mathscr{P})_{x}=\left\{P_{i}: i \leq k\right\}$, where $(\mathscr{P})_{x}=\{P \in \mathscr{P}: x \in P\}$. If each element of $(\mathscr{P})_{x}$ is not a sequential neighborhood of $x$ in $X$, then for each $i \leq k$, there exists a sequence $\left\{x_{i n}\right\}$ converging to $x$ such that $\left\{x_{i n}\right\}$ is not eventually in $P_{i}$. For each $n \in \mathbb{N}$ and $i \leq k$, put $y_{i+(n-1) k}=x_{i n}$, then $\left\{y_{m}\right\}$ converges to $x$ and is not eventually in each $P_{i}$, a contradiction. Thus there exists $P_{x} \in \mathscr{P}$ such that $P_{x}$ is a sequential neighborhood of $x$ in $X$. Put $\mathscr{F}=$ $\left\{P_{x}: x \in X\right\}$, then $\mathscr{F}$ is an $s n$-cover for $X$.

(3) $\Rightarrow(1)$. Suppose $\left\{\mathscr{P}_{n}\right\}$ is a weak development consisting of point-finite $s n$-covers for $X$. For each $i \in \mathbb{N}$, let $\mathscr{P}_{i}=\left\{P_{\alpha}: \alpha \in \Lambda_{i}\right\}$, endow $\Lambda_{i}$ with the discrete topology, then $\Lambda_{i}$ is a metric space. Put

$$
M=\left\{\alpha=\left(\alpha_{i}\right) \in \prod_{i \in \mathbb{N}} \Lambda_{i}:\left\langle P_{\alpha_{i}}\right\rangle \text { forms a network at some point } x_{\alpha} \text { in } X\right\}
$$

and endow $M$ with the subspace topology induced from the usual product topology of the collection $\left\{\Lambda_{i}: i \in \mathbb{N}\right\}$ of metric spaces, then $M$ is a metric space. Since $X$ is Hausdorff, $x_{\alpha}$ is unique in $X$. For each $\alpha \in M$, we define $f: M \rightarrow X$ by $f(\alpha)=x_{\alpha}$. For each $x \in X$ and $i \in \mathbb{N}$, there exists $\alpha_{i} \in \Lambda_{i}$ such that $x \in P_{\alpha_{i}}$. From $\left\{\mathscr{P}_{i}\right\}$ being a point-star network for $X,\left\{P_{\alpha_{i}}: i \in \mathbb{N}\right\}$ is a network of $x$ in $X$. Put $\alpha=\left(\alpha_{i}\right)$, then $\alpha \in M$ and $f(\alpha)=x$. Thus $f$ is surjective. Suppose $\alpha=\left(\alpha_{i}\right) \in M$ and $f(\alpha)=x \in U \in \tau(X)$, then there exists $n \in \mathbb{N}$ such that $P_{\alpha_{n}} \subset U$. Put

$$
V=\left\{\beta \in M \text { : the } n \text {th coordinate of } \beta \text { is } \alpha_{n}\right\} .
$$

Then $\alpha \in V \in \tau(M)$, and $f(V) \subset P_{\alpha_{n}} \subset U$. Hence $f$ is continuous.

For each $x \in X$ and $i \in \mathbb{N}$, put

$$
B_{i}=\left\{\alpha_{i} \in \Lambda_{i}: x \in P_{\alpha_{i}}\right\}
$$

then $\prod_{i \in \mathbb{N}} B_{i}$ is compact in $\prod_{i \in \mathbb{N}} \Lambda_{i}$. If $\alpha=\left(\alpha_{i}\right) \in \prod_{i \in \mathbb{N}} B_{i}$, then $\left\langle P_{\alpha_{i}}\right\rangle$ is a network of $x$ in $X$. So $\alpha \in M$ and $f(\alpha)=x$. Hence $\prod_{i \in \mathbb{N}} B_{i} \subset f^{-1}(x)$; If $\alpha=\left(\alpha_{i}\right) \in f^{-1}(x)$, then $x \in \bigcap_{i \in \mathbb{N}} P_{\alpha_{i}}$, so $\alpha \in \prod_{i \in \mathbb{N}} B_{i}$. Thus $f^{-1}(x) \subset \prod_{i \in \mathbb{N}} B_{i}$. Therefore, $f^{-1}(x)=\prod_{i \in \mathbb{N}} B_{i}$. This implies that $f$ is a compact mapping. 
We will prove that $f$ is weak-open. For each $x \in X$, since every $\mathscr{P}_{i}$ is an $s n$-cover for $X$, then there exists $\alpha_{i} \in \Lambda_{i}$ such that $P_{\alpha_{i}}$ is a sequential neighborhood of $x$ in $X$. From $\left\{\mathscr{P}_{i}\right\}$ a point-star network for $X,\left\langle P_{\alpha_{i}}\right\rangle$ is a network of $x$ in $X$. Put $\beta_{x}=\left(\alpha_{i}\right) \in \prod_{i \in \mathbb{N}} \Lambda_{i}$, then $\beta_{x} \in f^{-1}(x)$.

Let $\left\{U_{m \beta_{x}}\right\}$ be a decreasing neighborhood base of $\beta_{x}$ in $M$, and put

$$
\begin{gathered}
\mathscr{B}_{x}=\left\{f\left(U_{m \beta_{x}}\right): m \in \mathbb{N}\right\}, \\
\mathscr{B}=\bigcup\left\{\mathscr{B}_{x}: x \in X\right\},
\end{gathered}
$$

then $\mathscr{B}$ satisfies (1), (2) in Definition 1.1. Suppose $G$ is open in $X$. For each $x \in G$, from $\beta_{x} \in f^{-1}(x), f^{-1}(G)$ is an open neighborhood of $\beta_{x}$ in $M$. Thus $U_{m \beta_{x}} \subset f^{-1}(G)$ for some $m \in \mathbb{N}$, so $f\left(U_{m \beta_{x}}\right) \subset G$ and $f\left(U_{m \beta_{x}}\right) \in \mathscr{B}_{x}$. On the other hand, suppose $G \subset X$ and for $x \in G$, there exists $B \in \mathscr{B}_{x}$ such that $B \subset G$. Let $B=f\left(U_{m \beta_{x}}\right)$ for some $m \in \mathbb{N}$, and let $\left\{x_{n}\right\}$ be a sequence converging to $x$ in $X$. Since $P_{\alpha_{i}}$ is a sequential neighborhood of $x$ in $X$ for each $i \in \mathbb{N}$, then $\left\{x_{n}\right\}$ is eventually in $P_{\alpha_{i}}$. For each $n \in \mathbb{N}$, if $x_{n} \in P_{\alpha_{i}}$, let $\alpha_{i n}=\alpha_{i}$; if $x_{n} \notin P_{\alpha_{i}}$, pick $\alpha_{i n} \in \Lambda_{i}$ such that $x_{n} \in P_{\alpha_{i} n}$. Thus there exists $n_{i} \in \mathbb{N}$ such that $\alpha_{i n}=\alpha_{i}$ for all $n>n_{i}$. So $\left\{\alpha_{i n}\right\}$ converges to $\alpha_{i}$. For each $n \in \mathbb{N}$, put

$$
\beta_{n}=\left(\alpha_{i n}\right) \in \prod_{i \in \mathbb{N}} \Lambda_{i}
$$

then $f\left(\beta_{n}\right)=x_{n}$ and $\left\{\beta_{n}\right\}$ converges to $\beta_{x}$. Since $U_{m \beta_{x}}$ is an open neighborhood $\beta_{x}$ in $M$, then $\left\{\beta_{n}\right\}$ is eventually in $U_{m \beta_{x}}$, so $\left\{x_{n}\right\}$ is eventually in $G$. Hence $G$ is a sequential neighborhood of $x$. So $G$ is sequential open in $X$. By $X$ being a sequential space, $G$ is open in $X$. This implies $\mathscr{B}$ is a weak base for $X$.

By the idea of $\mathscr{B}, f$ is weak-open.

We give examples illustrating Theorem 2.1 of this note.

Example 2.2. Let $X$ be the Arens space $S_{2}$ (see [6, Example 1.8.6]). It is not difficult to see that the space is a weak-open compact image of a metric space. But $X$ is not an open compact image of a metric space, because $X$ is not developable. Thus the following holds.

A weak-open compact image of a metric space is not always an open compact image of a metric space.

Example 2.3. Let $Y$ be the weak Cauchy space in [10, Example 2.14(3)]. By the construction, $Y$ is a quotient compact image of a metric space. But $Y$ is not Cauchy, $Y$ is not a weak-open compact image of a metric space by Theorem 2.1. Thus the following holds:

A quotient compact image of a metric space is not always a weak-open compact image of a metric space.

\section{Acknowledgments}

This work is supported by the NSF of Hunan Province in China (no. 05JJ40103) and the NSF of Education Department of Hunan Province in China (no. 03C204). 


\section{References}

[1] A. V. Arhangel'skiŭ, Mappings and spaces, Russian Mathematical Surveys 21 (1966), no. 4, 115162.

[2] S. P. Franklin, Spaces in which sequences suffice, Fundamenta Mathematicae 57 (1965), 107-115.

[3] Y. Ikeda, C. Liu, and Y. Tanaka, Quotient compact images of metric spaces, and related matters, Topology and Its Applications 122 (2002), no. 1-2, 237-252.

[4] Z. Li and S. Lin, On the weak-open images of metric spaces, Czechoslovak Mathematical Journal 54 (2004), no. 2, 393-400.

[5] S. Lin, On the quotient compact images of metric spaces, Advances in Mathematics 21 (1992), no. 1, 93-96.

[6] __ Generalized Metric Spaces and Mappings, Chinese Scientific, Beijing, 1995.

[7] _ Point-Countable Coverings and Sequence-Covering Mappings, Chinese Distinguished Scholars Foundation Academic Publications, Chinese Scientific, Beijing, 2002.

[8] S. Lin, Y. C. Zhou, and P. F. Yan, On sequence-covering $\pi$-mappings, Acta Mathematica Sinica 45 (2002), no. 6, 1157-1164.

[9] F. Siwiec, On defining a space by a weak base, Pacific Journal of Mathematics 52 (1974), 233-245.

[10] Y. Tanaka, Symmetric spaces, $g$-developable spaces and g-metrizable spaces, Mathematica Japonica 36 (1991), no. 1, 71-84.

[11] Y. Tanaka and Z. Li, Certain covering-maps and k-networks, and related matters, Topology Proceedings 27 (2003), no. 1, 317-334.

[12] S. Xia, Characterizations of certain g-first countable spaces, Advances in Mathematics 29 (2000), no. $1,61-64$.

Zhiyun Yin: Department of Information, Hunan Business College, Changsha, Hunan 410205, China

E-mail address: yzy8846@163.com 


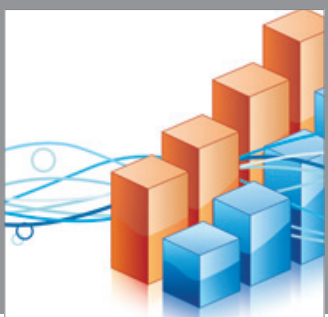

Advances in

Operations Research

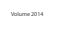

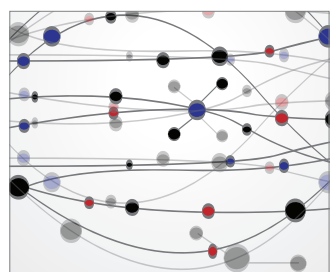

\section{The Scientific} World Journal
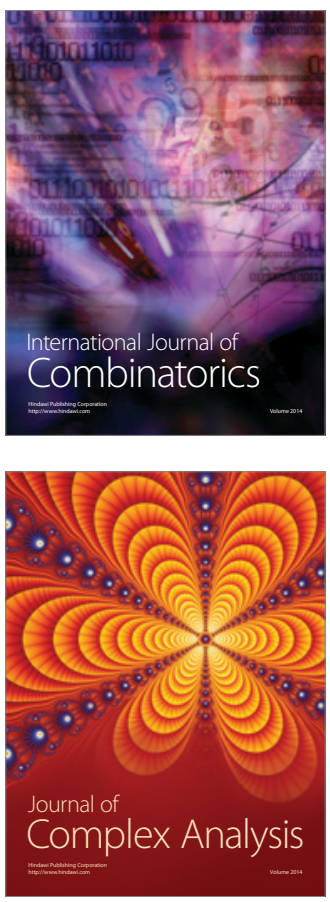

International Journal of

Mathematics and

Mathematical

Sciences
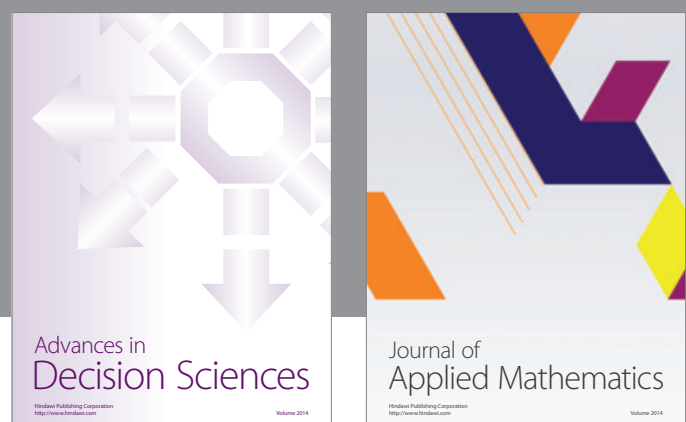

Journal of

Applied Mathematics
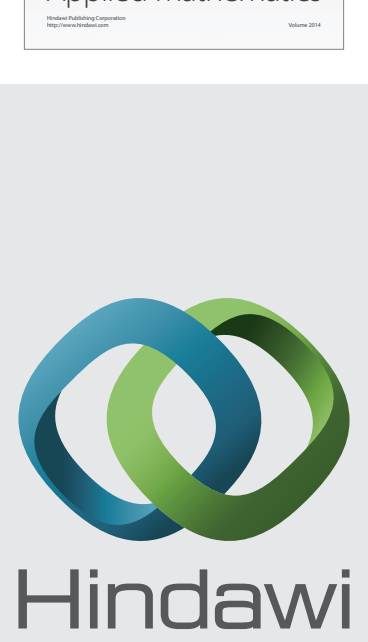

Submit your manuscripts at http://www.hindawi.com
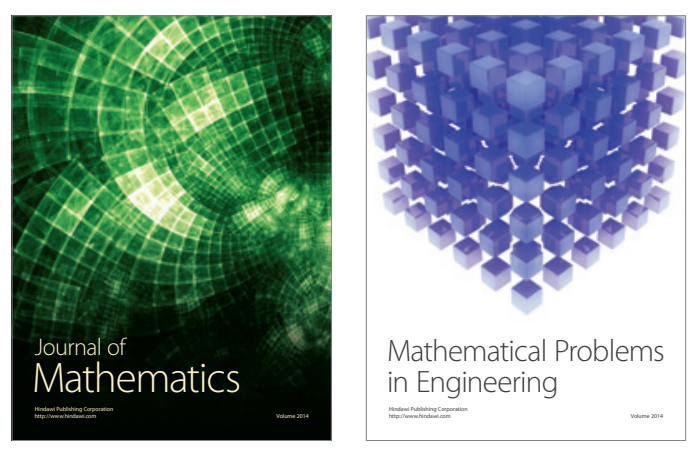

Mathematical Problems in Engineering
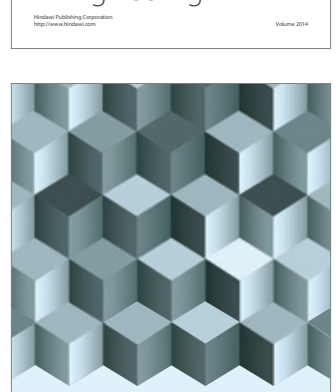

Journal of

Function Spaces
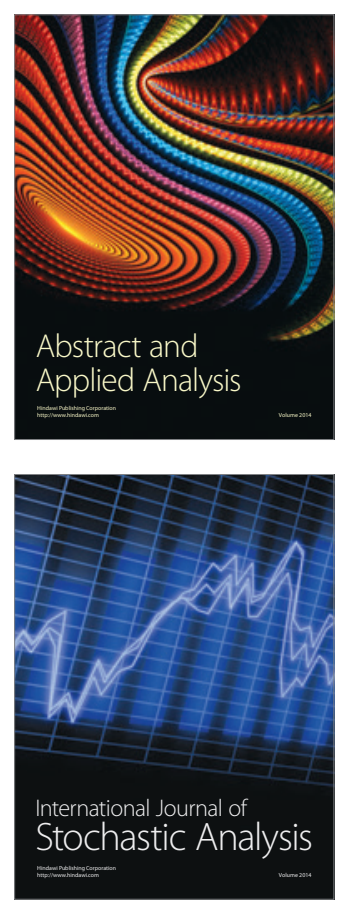

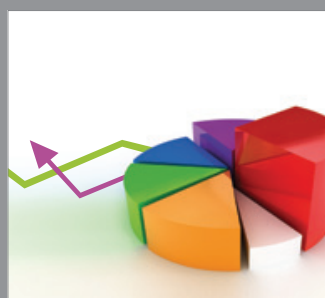

ournal of

Probability and Statistics

Promensencen
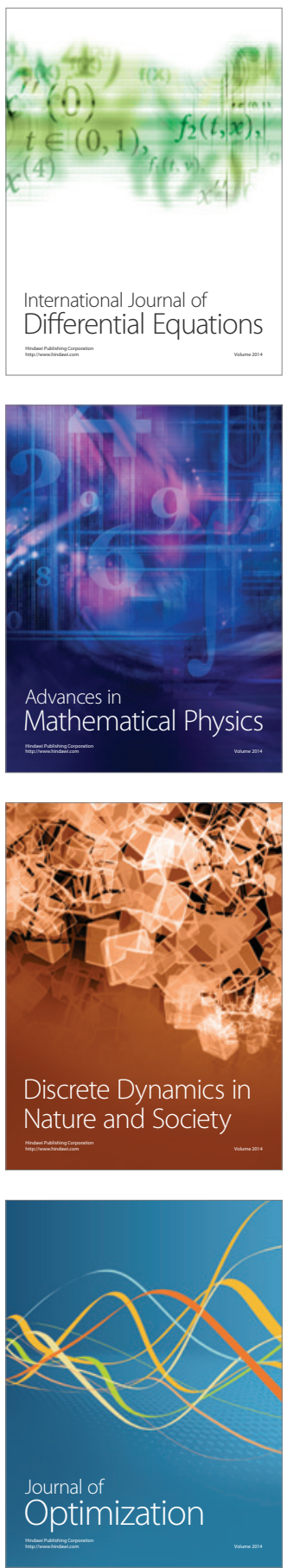